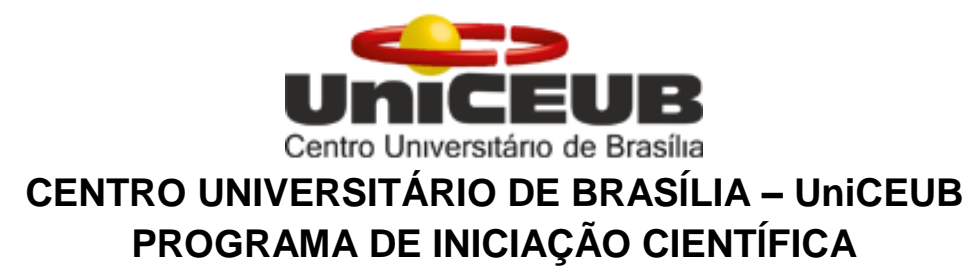

Luiz Matheus Xavier Cocentino

Natália Ramires Kairala

\title{
“O IMPACTO NA DIMINUIÇÃO DO TEMPO DE VENTILAÇÃO MECÂNICA APÓS A IMPLANTAÇÃO DE UM PROTOCOLO DE EXTUBAÇÃO PROGRAMADA (CHECK LIST) EM RECEM NASCIDOS EM UMA UNIDADE DE TERAPIA INTENSIVA NEONATAL. "
}




\title{
UnTC=Uв \\ Centro Unıversitárıo de Brasílıa
}

\author{
Luiz Matheus Xavier Cocentino \\ Natália Ramires Kairala
}
“O IMPACTO NA DIMINUIÇÃO DO TEMPO DE VENTILAÇÃO MECÂNICA APÓS A IMPLANTAÇÃO DE UM PROTOCOLO DE EXTUBAÇÃO PROGRAMADA (CHECK LIST) EM RECEM NASCIDOS EM UMA UNIDADE DE TERAPIA INTENSIVA NEONATAL. "

\author{
Relatório final de pesquisa de Iniciação Científica \\ apresentado à Assessoria de Pós-Graduação e Pesquisa. \\ Orientação: Andréa Lopes Ramires Kairala.
}

\section{BRASÍLIA}


Dedico este trabalho primeiramente à Deus, por ter me abençoado e ter me dado força para chegar aonde estou chegando neste momento. Agradeço também à minha companheira e à minha filha, companheiras de todas as horas, fontes de inspiração, energia e alegria nesse árduo caminho. Por último, agradeço infinitamente aos meus pais, por todo esforço, suporte emocional e financeiro, sem vocês não estaria aonde estou agora, vocês são o meu espelho de ser humano para a vida. Amo todos vocês. 


\section{Agradecimentos}

Agradeço à minha orientadora, Professora Dra. Andréa Lopes Ramires Kairala, pela paciência, dedicação e ensinamentos que possibilitaram que eu realizasse esse trabalho, saiba que você é muito querida e um exemplo de médica para mim.

Agradeço também a todos que contribuíram para a realização deste trabalho, seja de forma direta ou indireta, fica registrado aqui, o meu muito obrigado. 


\section{LISTA DE TABELAS}

Tabela 01 - Relação de RN por período de estudo...........................................................

Tabela 02 - Perfil de RN de acordo com o peso ao nascer....................................................9

Tabela 03 - Patologias que levaram à internação.................................................................10

Tabela 04 - Tempo de permanência em VM...................................................................10

Tabela 05 - Uso de corticoide prévio a extubação do RN...................................................11

Tabela 06 - Número de falhas de extubação de acordo com o período de estudo....................11

Tabela 07 - Relação entre a idade gestacional e falhas de extubação.....................................12

Tabela 08 - Relação do peso de nascimento com falhas de extubação....................................12

Tabela 09 - Relação entre as patologias dos RN com falhas de extubação..............................13

Tabela 10 - Relação entre o tempo de VM com falhas de extubação......................................14

Tabela 11- Relação entre falha no TRE com falhas de extubação....................................... 14

Tabela 12 - Relação entre o pico de pressão com falhas de extubação....................................14 


\section{LISTA DE ABREVIATURAS}

DF - Distrito Federal

DMH - Doença da Membrana Hialina

FIO2 - Fração Inspirada de Oxigênio

FR - Frequência Respiratória

G - Grama

PaCO2: Pressão Arterial de Dióxido de Carbono

PaO2: Pressão Arterial de Oxigênio

PMPB - Prematuro Muito Baixo Peso

PP - Pressão de Pico

RN - Recém-Nascido

RNT - Recém-Nascido de Termo

RNPT - Recém-Nascido Pré Termo

SatO2 - Saturação de Oxigênio

TRE - Teste de Respiração Espontânea

UTI - Unidade de Terapia Intensiva

UTIN - Unidade de Terapia Intensiva Neonatal

VM - Ventilação Mecânica

VMI - Ventilação Mecânica Invasiva 


\section{SUMÁRIO}

1. INTRODUÇÃO

2. OBJETIVOS

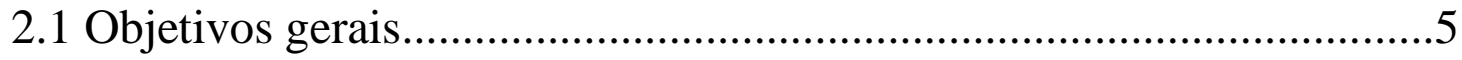

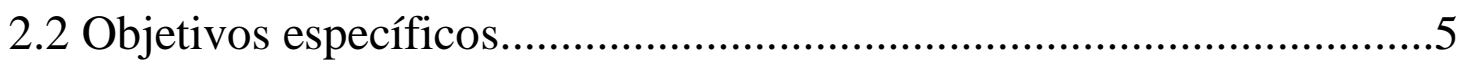

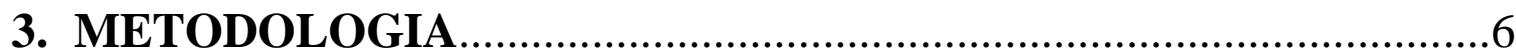

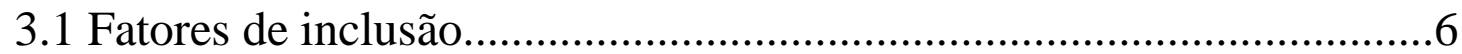

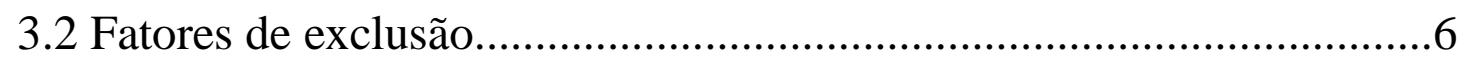

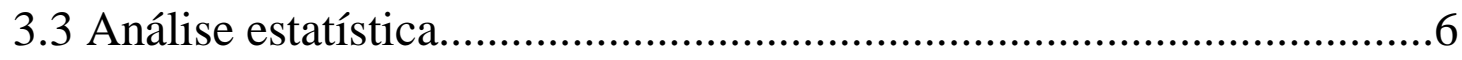

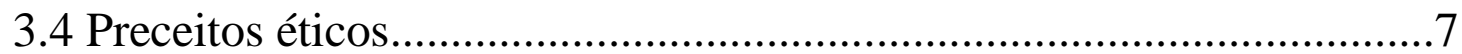

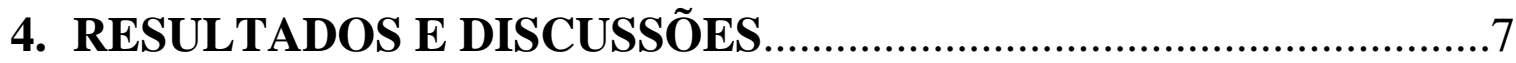

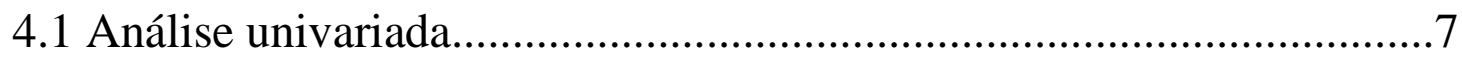

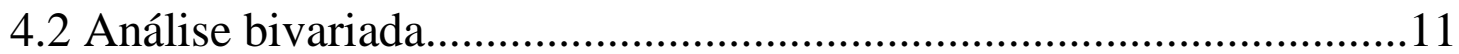

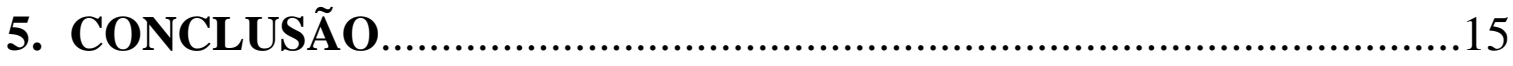

REFERÊNCIAS

ANEXO 1

ANEXO 2 


\section{RESUMO}

INTRODUÇÃO: A ventilação mecânica invasiva (VMI) é um procedimento de fundamental importância em unidades de Terapia Intensiva, com maior destaque nas Unidades de Terapia Intensiva Neonatal (UTIN). A ventilação mecânica (VM) é um procedimento necessário para a sobrevivência, mas não é isento de riscos, causando lesões ao pulmão doente ou imaturo. A forma de executar o processo de desmame é um dos momentos cruciais no uso da ventilação pulmonar mecânica que pode influenciar diretamente no seu sucesso ou insucesso. OBJETIVO: avaliar a efetividade da implantação de um protocolo de extubação programada (checklist), na diminuição do tempo de ventilação mecânica em recém-nascidos (RN) da Unidade de Terapia Intensiva Neonatal de um hospital escola privado do Distrito Federal (DF). METODOLOGIA: Análise quantitativa, descritiva e retrospectiva de prontuário, com levantamento de dados dos RN internados na UTIN de um hospital privado do DF no período de janeiro de 2013 a dezembro de 2017, durante o qual foram submetidos à VM e ao procedimento de extubação. RESULTADOS: O número de pacientes internados na UTIN no período do estudo (entre 2013 e 2017) foi 1569. O número de RN internados na UTIN logo após o nascimento e que necessitaram de VM foi 370. A amostra após seleção dos pacientes de acordo com os critérios de inclusão e exclusão foi $\mathrm{N}=124 \mathrm{RN}$. O número de pacientes internados por ano de nascimento foi 2013: 22 (17,7\%) pacientes, 2014: 24 (19,3\%), 2015: 21(16,9\%), 2016: 25 (20,1\%) e 2017: 32 (25,8\%). Foi observado uma taxa de falha de extubação de 42,86 $\%$ antes da implantação do protocolo, contra 35,71 \% após sua consolidação. CONCLUSÃO: O processo de retirada da VM, na grande maioria dos serviços de terapia intensiva neonatal, ainda está sujeito a condutas pouco embasadas cientificamente. Sob o ponto de vista profissional, criar mecanismos facilitadores na UTI pode abreviar o tempo de VM; visto que os profissionais devem se sentir mais seguros ao identificar o paciente capaz de se submeter ao teste de respiração espontânea (TRE) diminuindo, assim, o grau de insucesso das extubações, garantindo melhor evolução do paciente.

Palavras - chave: Ventilação Mecânica. Extubação. Neonatologia 


\begin{abstract}
INTRODUCTION: Invasive mechanical ventilation (IMV) is a procedure of fundamental importance in Intensive Care Units, most prominently in Neonatal Intensive Care Units (NICUs). Mechanical ventilation (MV) is a necessary procedure for survival, but is not free of risks, causing injury to the diseased or diseased lung. The way to perform the weaning process is one of the crucial moments in the use of mechanical pulmonary ventilation that can directly influence your success or failure. OBJECTIVE: to evaluate the effectiveness of the implantation of a programmed extubation protocol (checklist), to reduce the mechanical ventilation time in newborns (NB) of the Neonatal Intensive Care Unit of a private school hospital in the Federal District. MATERIALS AND METHODS: Quantitative, descriptive and retrospective analysis of medical records, with data from NBs admitted to the NICU of a private hospital in the Federal District from January 2013 to December 2017, during which they were submitted to MV and extubation procedure. RESULTS: The number of patients admitted to the neonatal ICU during the study period (between 2013 and 2017) was 1569. The number of infants admitted to the NICU soon after birth and who required MV was 370. The sample after selection of patients according to the inclusion criteria and exclusion was $\mathrm{N}=124 \mathrm{RN}$. The number of hospitalized patients per year of birth was 2013: 22 (17.7\%) patients, 2014: 24 (19.3\%), 2015: 21 (16.9\%), 2016: 25 (20.1\%) and 2017: 32 (25.8\%). An extubation failure rate of $42.86 \%$ was observed before implantation of the protocol, compared to $35.71 \%$ after its consolidation. CONCLUSIONS: The process of withdrawal of MV, in the great majority of neonatal intensive care services, is still subject to conduct that is not scientifically based. From the professional point of view, creating facilitative mechanisms in the ICU can shorten MV time; since the professionals must feel more confident in identifying the patient capable of undergoing the spontaneous breathing test (ERT), thus reducing the degree of failure of the extubations, thus ensuring a better evolution of the patient.
\end{abstract}

Key - Word: Mechanical Ventilation. Extubation. Neonatology 


\section{INTRODUÇÃO}

A ventilação mecânica invasiva (VMI) é um procedimento de fundamental importância em unidades de Terapia Intensiva, com maior destaque nas Unidades de Terapia Intensiva Neonatal (UTIN), em que seu uso atinge cifras superiores a 60\% em Prematuros de Muito Baixo Peso (PMBP). (STOLL,2010; KARCZ, 2011)

As patologias respiratórias são frequentes em UTIN, causando danos pulmonares que levam o recém-nascido a necessitar de ventilação mecânica invasiva (VMI) como forma de tratamento. Esse fato está relacionado a mudanças na fisiologia respiratória que ocorrem algumas horas antes do nascimento e às características próprias do sistema respiratório especialmente em recém nascidos prematuros (RNPT) tais como: vias aéreas mais estreitas, menor número de alvéolos, deficiência de ventilação colateral, o que predispõe ao colapso alveolar e à diminuição ou ausência de surfactante, o que provoca uma maior instabilidade das vias aéreas superiores e da caixa torácica, dificultando a realização de um processo de extubação bem-sucedido e, por consequência, na necessidade de uma reintubação.(SANTOS, OLIVEIRA, BERENCHTEIN,2014; HERMETO , 2009)

Distúrbios como Membrana Hialina, Síndrome da Aspiração de Mecônio, Apneia da Prematuridade, Taquipneia Transitória do RN, Pneumonia, Hipertensão Pulmonar Persistente, Displasia Broncopulmonar e Persistência do Ducto Arterioso são frequentes na UTIN e danificam o pulmão o que leva o RN a necessitar de VMI. (AZEREDO,2002)

A VM é um procedimento necessário para a sobrevivência, mas não é isento de riscos podendo causar lesões ao pulmão doente ou imaturo. Em neonatos, o uso da VM por tempo excessivo e as vezes desnecessário pode levar à várias complicações como: infecção, displasia broncopulmonar, maior risco de morte e pior prognóstico de desenvolvimento neuropsicomotor. (ANTUNES, 2012)

Os principais fatores que estão associados à falha na extubação em RN são:

- Pulmonares: doença pulmonar primária não resolvida, atelectasia pós extubação, insuficiência respiratória da prematuridade, displasia broncopulmonar, paralisia do diafragma;

- Vias aéreas superiores: edema e/ou excesso de secreção traqueal, estenose subglótica, laringotraqueomalácia, traqueobronquite necrosante; 
- Cardiovasculares: persistência do ducto arterioso, sobrecarga de líquidos, cardiopatia congênita com sobrecarga pulmonar;

- Sistema Nervoso Central: apneia no recém-nascido muito prematuro, hemorragia intraventricular, dano cerebral por hipóxia-isquêmica, por drogas como fenobarbital;

- Miscelâneas: sem diagnóstico reconhecido sepse, alterações metabólicas. (GOLDSMITH, SHARP,1988)

A classificação dos recém-nascidos pré-termo (RNPT) e recém-nascido de termo (RNT) segundo a Sociedade Brasileira de Pediatria (SBP) é:

- Prematuridade extrema $\mathrm{RN}<28$ semanas;

- Prematuridade grave RN com idade gestacional entre 28 a 30 semanas;

- Prematuridade moderada RN com idade gestacional entre 31 a 33 semanas;

- Prematuridade tardia RN com idade gestacional entre 34 a 36 semanas;

- Recém-nascido de termo com idade gestacional entre 37 a 40 semanas.

Essa classificação é de extrema relevância para determinar o risco quanto à evolução dos $\mathrm{RN}$, levando em consideração que as patologias que levam a insuficiência respiratória são diferentes nesses grupos.

O processo de desmame, período de transição da ventilação mecânica para a espontânea nos pacientes que permaneceram com suporte ventilatório por tempo superior ao período de 24 horas, deve ser realizado assim que o RN se estabilize da causa que indicou o procedimento, fazendo-se necessário que o suporte seja o mais breve possível, a fim de minimizar complicações. (PEREIRA, SMITH, HENRY, 2007) Esteban et al (1994), estimaram que um paciente em VM passa até $42 \%$ do tempo no processo de desmame

A forma de executar o processo de desmame é um dos momentos cruciais no uso da ventilação pulmonar mecânica que pode influenciar diretamente no seu sucesso ou insucesso. A finalização de todo este processo resulta na retirada da cânula oro-traqueal, denominada extubação. Nos RNPT, a extubação precoce diminui os efeitos adversos da ventilação mecânica, em contrapartida é um procedimento delicado que possui seus próprios riscos ao paciente como, por exemplo, perda da proteção das vias aéreas, fadiga e sobrecarga muscular; estima-se que um terço dos pacientes necessite de reintubação. (SANTOS, OLIVEIRA, BERENCHTEIN, 2014; HERMETO, 2009; GOLDWASSER, FARIAS, 2007; GOLDWASSER, DAVID, 2007; BOUSSO,2002) 
No contexto de uma unidade de terapia intensiva (UTI) os profissionais devem estar aptos a identificar o paciente capaz de ser submetido à extubação, abreviando o tempo de ventilação mecânica, evitando o insucesso da extubação e garantindo melhor evolução do paciente. (HERMETO,2009). O sucesso do desmame depende, dentre outros, da presença de um drive respiratório eficaz, uma força muscular respiratória adequada e de uma magnitude de resistência à carga respiratória. (GOLDWASSER, FARIAS, 2007; GOLDWASSER, DAVID,2007)

A falha de extubação é a necessidade de reintubação e retorno ao suporte ventilatório sendo considerada precoce quando ocorre em menos de $24 \mathrm{~h}$ após a extubação. (HARRISON,2002). A fim de se minimizar as taxas de reintubação, existem diversas estratégias e critérios preditivos que podem auxiliar na avaliação e no sucesso do desmame e da extubação. (GONÇALVES, 2007)

O termo interrupção da ventilação mecânica refere-se aos pacientes que toleraram um teste de respiração espontânea e que podem ou não ser elegíveis para extubação. (TEIXEIRA, MACCARI, VIEIRA, et al,2012)

Define-se sucesso da interrupção da ventilação mecânica como um teste de respiração espontânea bem-sucedido. Os pacientes que obtiverem sucesso devem ser avaliados quanto à indicação de retirada da via aérea artificial. Quando o paciente não tolera o teste de respiração espontânea, considera-se fracasso na interrupção da ventilação mecânica. No caso de fracasso o paciente deverá receber suporte ventilatório que promova repouso da musculatura. Uma revisão das possíveis causas desse fracasso deverá ser feita pela equipe assistente, bem como o planejamento da estratégia a ser adotada a seguir, nova tentativa de interrupção da ventilação mecânica ou desmame gradual. (TEIXEIRA, MACCARI, VIEIRA, et al,2012).

Danaga et al (2009), afirmam que o uso de protocolos é de grande importância para evitar o insucesso do desmame, pois as reintubações ocorrem em cerca de $20 \%$ das extubações e está associada a um maior risco de pneumonia hospitalar, tempo prolongado na ventilação mecânica e maior permanência na UTI. Colombo et al (2007), afirmam que a implementação de um protocolo de desmame de ventilação atende melhor os requisitos de eficiência e segurança do procedimento de extubação, favorecendo a evolução e diminuindo as complicações.

Estudos têm sido realizados em busca do melhor parâmetro para prever a falha ou o sucesso na extubação (ANDRADE,2010). A possibilidade do desmame em pacientes submetidos a ventilação mecânica tem sido definida utilizando sinais e sintomas clínicos, o que reflete em julgamentos e estilos individualizados. Este empirismo aplicado ao desmame pode 
prolongar o tempo de ventilação mecânica, conforme comprovam estudos randomizados e controlados (GONÇALVES,2007; ULSENHEIMER,2006). Por outro lado, estabelecer estratégias como protocolos que buscam objetivar e agrupar condições clínicas a favor da extubação colabora na identificação sistemática dos pacientes que podem ser submetidos ao processo de desmame.

No trabalho de Ulsenheimer (2006) a fim de avaliar a eficácia do uso de um protocolo implantado, foi realizado um estudo retrospectivo com 75 casos de crianças submetidas a ventilação mecânica, comparando crianças extubadas seguindo o protocolo e crianças extubadas de outra forma. O resultado demonstrou que o uso do protocolo reduziu tempo de internação, tempo de desmame e o número de reintubações, sendo eficaz e seguro.

Teixeira et al (2012) concluiu que o uso do protocolo somados a parâmetro clínico do paciente são fundamentais para o sucesso de um processo de extubação. O estudo observou uma amostra de 731 pacientes de difícil desmame (falha no primeiro teste de ventilação espontânea) desses, 533 (72,9\%) foram incluídos no grupo protocolo e 198 foram incluídos no grupo não protocolo. Observou-se que a taxa de falha de extubação no grupo não protocolo foi significativamente maior que no grupo protocolo (30,4\% vs. $13,3 \%$;).

Apesar de protocolos e experiências de vários serviços, a falha na extubação tem ocorrido em torno de $24 \%$ dos casos. Por isso, alguns índices e parâmetros são utilizados para prever e identificar o momento adequado para uma extubação, estes, incluem diferentes funções fisiológicas do sistema respiratório, que permitem a identificação do momento em que o paciente é capaz de assumir e manter a sua ventilação, evitando a ventilação por tempo prolongado e suas complicações. (GOLDWASSER, FARIAS, FREITAS et al, 2007)

Algumas condições clínicas e laboratoriais são conhecidas para que se possa considerar o RN apto à extubação: frequência respiratória adequada, ausência de utilização de musculatura acessória, ausência de batimentos de asa de nariz, estabilidade hemodinâmica e ausência de crises convulsivas, além de adequada oxigenação (PaO2/Fi02>200), saturação de oxigênio (Sat02>93\%) e pressão arterial de dióxido de carbono ( $\mathrm{PaC} 02<45 \mathrm{mmHg}$ ). (HERMETO, 2009)

Estabelecer estratégias para identificar sistematicamente os pacientes que podem ser submetidos ao processo de desmame torna os profissionais de saúde mais aptos e seguros, abreviando assim o tempo de uso de ventilação mecânica. (GONÇALVES,2007; ULSENHEIMER, 2006)

O protocolo de extubação utilizado na Unidade de Terapia Intensiva Neonatal (UTIN) do presente estudo foi implementado pelo serviço no mês de março de 2014, com a 
utilização de um checklist de extubação (ANEXO 1) preenchido em conjunto por médicos e fisioterapeutas, que define como critérios: estabilidade hemodinâmica, reversibilidade total ou parcial da causa que motivou a VM, estabilidade metabólicas e hematológicas, condições neurológicas adequadas, parâmetros ventilatórios: $\mathrm{FR} \leq 60 \mathrm{rpm}, \mathrm{PEEP} \leq 5, \mathrm{FiO}^{2} \leq 30 \%$, exames complementares: relação $\mathrm{PaO}^{2} / \mathrm{FiO}^{2} \geq 200$, com todos os parâmetros adequados; realização de fisioterapia respiratória 1 hora antes da Extubação, realizar o teste de respiração espontânea (TRE) em modo PSV (Ventilação com Pressão de Suporte) por pelo menos 15 minutos. O protocolo foi implementado como ferramenta de avaliação do desmame da VM nos RecémNascidos internados na UTIN, bem como uma maneira de diminuir o número de falhas de extubações.

\section{OBJETIVOS}

\subsection{OBJETIVOS GERAIS:}

Avaliar a efetividade da implantação de um protocolo de extubação programada (checklist), na diminuição do tempo de ventilação mecânica em RN da Unidade de Terapia Intensiva Neonatal de um hospital escola privado do DF.

\subsection{OBJETIVOS ESPECÍFICOS:}

1. Analisar a efetividade e dificuldades da implantação de um protocolo de extubação programada, no período de um ano antes de sua implantação (2013), no ano de implantação (março de 2014) e no período de consolidação (2015/2016) em RN de uma UTI Neonatal.

2. Avaliar a prevalência de falhas de extubação antes e após a implantação do protocolo na UTIN.

3. Relacionar as possíveis causas do insucesso da retirada da VM, através da análise do perfil dos RN internados na UTIN.

4. Identificar os critérios incluídos no checklist que mais contraindicaram a extubação.

5. Identificar os critérios incluídos no checklist mais relacionados com a falha de extubação.

\section{METODOLOGIA}


Análise quantitativa, descritiva e retrospectiva de prontuário, com levantamento de dados dos RN internados na UTIN de um hospital privado do DF no período de janeiro de 2013 a dezembro de 2017, durante o qual foram submetidos à VM e ao procedimento de extubação. Como instrumento de coleta foi utilizado uma ficha, com as seguintes variáveis: ano de nascimento, idade gestacional ao nascimento e a idade corrigida no momento da extubação, peso ao nascimento, classificação do RN, APGAR, patologia que levou a indicação de VM, tempo de VM, uso de corticoide, Fração inspirada de Oxigênio (FiO2), pressão de pico (PP), frequência respiratória no momento da extubação (FR), realização ou não do teste de respiração espontânea (TRE), falha de extubação e SNAP PE II (Vide anexo 2).

\subsection{Fatores de Inclusão}

- Todos os recém-nascidos de termo (RNT) e RNPT nascidos e internados na UTIN e que necessitaram de Ventilação Mecânica Invasiva (VMI), por mais de 24 horas, no período entre janeiro de 2013 e dezembro de 2017.

\subsection{Fatores de exclusão:}

- RN que permaneceram entubados por menos de 24 horas;

- RN que sofreram extubação acidental;

- RN que foram a óbito no período entre o desmame e extubação;

- RN com malformação congênita, síndromes genéticas ou complicações cardíacas;

- RN que não nasceram no Hospital Santa Marta (HSM) e que vieram transferidos de outro centro médico;

- RN com prontuário incompleto.

\subsection{Análises Estatísticas}

Para as análises estatísticas foram utilizados os Softwares SPSS. Realizada análise descritiva das variáveis com média, desvio padrão e porcentagem. O Teste Quiquadrado foi usado para verificar a existência de associação estatisticamente significante entre o sucesso e a falha na extubação no período antes e após a implantação de protocolo de extubação na UTIN. O critério de determinação significativo considerado será p<0,05.

Um levantamento bibliográfico e revisão de literatura das pesquisas e assuntos mais recentes na área através de bases de dados automatizadas como Bireme, Scielo, Pubmed foi 
realizado nessa etapa de análise de dados, visando a comparação dos resultados obtidos na pesquisa com os dados existentes na literatura.

\subsection{Preceitos éticos}

O projeto atende os requisitos éticos e os pesquisadores foram considerados aptos a iniciar a coleta de dados. Considerações finais a critério do CEP: protocolo aprovado ad referendum pelo CEP-UniCEUB, com parecer $\mathrm{N}^{\circ}$ 2.994.102/18

\section{RESULTADOS E DISCUSSÃO}

\subsection{ANÁLISE UNIVARIADA}

O número de pacientes internados na UTI neonatal no período do estudo (entre 2013 e 2017) foi 1569. O número de RN internados na UTIN logo após o nascimento e que necessitaram de VM foi 370. A amostra após seleção dos pacientes de acordo com os critérios de inclusão e exclusão foi $\mathrm{N}=124 \mathrm{RN}$. O número de pacientes internados por ano de nascimento foi 2013: 22(17,7\%) pacientes, 2014: 24 (19,3\%), 2015: 21(16,9\%), 2016: $25(20,1 \%)$ e 2017: 32 $(25,8 \%)$. (Gráfico 1)

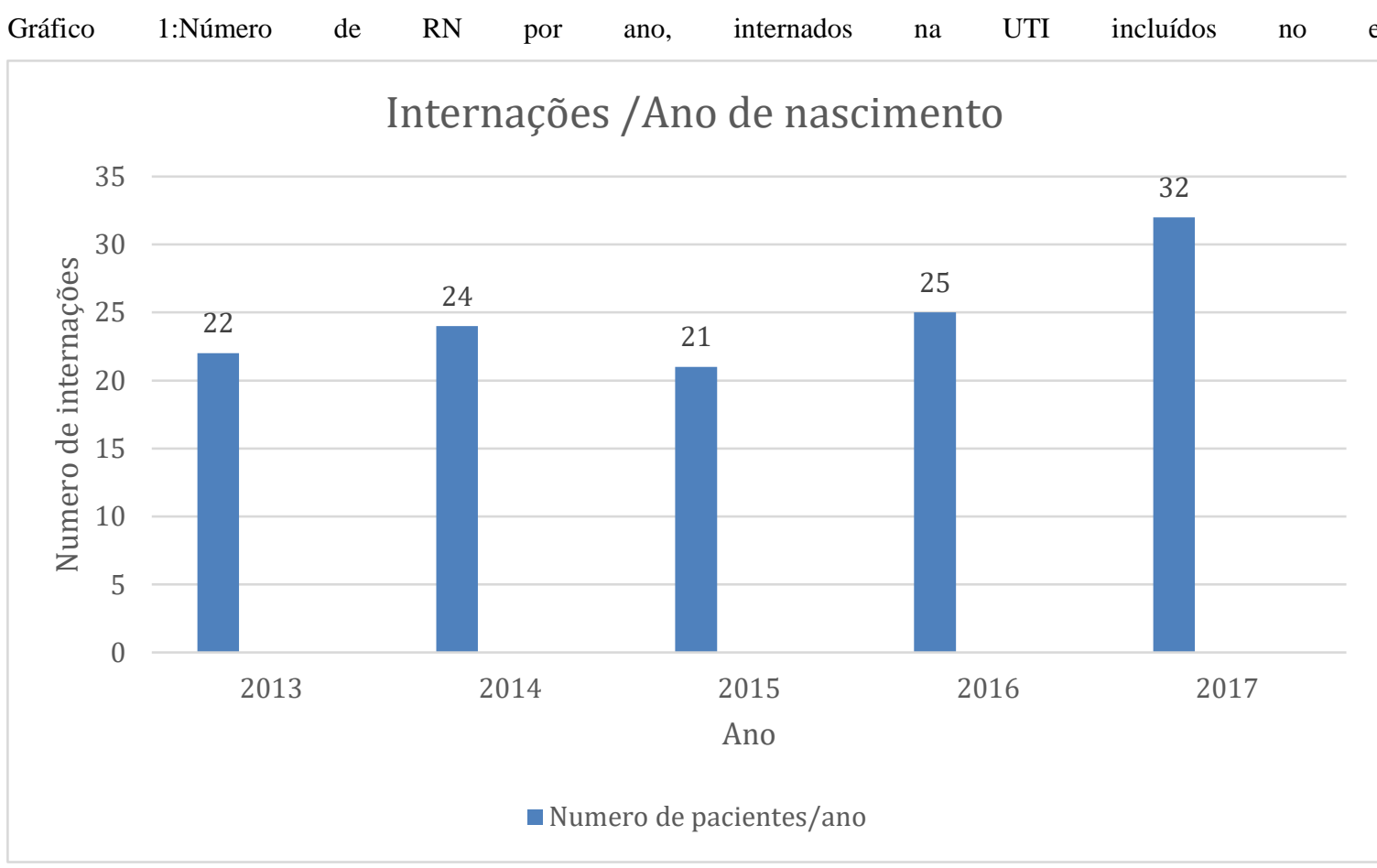

Fonte: autoria própria.

Foi observado $11,3 \%(\mathrm{~N}=14)$ falhas de extubação.

O período do estudo foi dividido em 3 intervalos de tempo (Tabela 1): 
1) Período antes da implantação do protocolo de extubação, que incluiu 27 pacientes, em um período de 14 meses.

2) Período considerado o ano da implantação do protocolo com um total de 18 pacientes, em um período de 12 meses.

3) Período de consolidação com 79 pacientes, com um período de 33 meses.

Tabela 1. Relação de RN por períodos do estudo.

\begin{tabular}{|c|c|c|c|}
\hline Período & $\mathbf{N}^{\circ}$ de $\mathbf{R N}$ & $\%$ & $\mathrm{~N}^{\circ}$ de meses \\
\hline $\begin{array}{lll}\text { Período antes } & \text { da } \\
\text { implantação } & & \end{array}$ & 27 & 21,7 & 14 \\
\hline Período de implantação & 18 & 14,5 & 12 \\
\hline Período de consolidação & 79 & 63,7 & 33 \\
\hline
\end{tabular}

Fonte: autoria própria.

Perfil do RN de acordo com a idade gestacional ao nascer. Foi observado que $71 \%$ dos recémnascidos eram muito prematuros ou prematuro extremo. (Figura 1)

Figura 01: perfil de RN de acordo com a idade gestacional.

\section{Idade Gestacional $(\mathrm{N}=124)$}

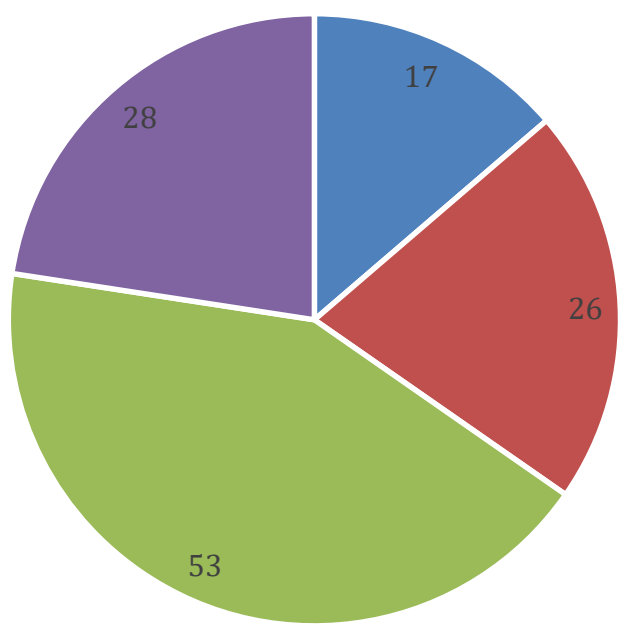

- RNT

- RNPT tardio

- Muito prematuro

- RNPT extremo 
Perfil do RN de acordo com o peso ao nascer: dos RN que necessitaram de VM, 81,5\% eram baixo peso (peso $<2.500 \mathrm{~g}$ ). (Tabela 2 ).

Tabela 02: perfil de RN de acordo com o peso ao nascer.

\begin{tabular}{l|l|l}
\hline Peso ao nascer & $\mathbf{N}^{\mathbf{0}}$ & $\%$ \\
\hline 2.500 a $3.800 \mathrm{~g}$ & 23 & 18,5 \\
\hline 1.500 a $2.500 \mathrm{~g}$ & 46 & 37,1 \\
\hline 1.000 a $1.500 \mathrm{~g}$ & 33 & 26,6 \\
\hline$<1.000 \mathrm{~g}$ & 22 & 17,7 \\
\hline Total & 124 & 100,0 \\
\hline
\end{tabular}

Fonte: autoria própria.

A análise das patologias que levaram a internação do RN e a necessidade de VM mostrou que em 74,1\% dos casos, a internação e o motivo de intubação foram relacionados com o aparelho respiratório e insuficiência respiratória; apenas $25,8 \%$ estavam relacionados com alterações hemodinâmicas como a sepse. (Tabela 03)

Tabela 03: Patologias que levaram a internação do RN.

\begin{tabular}{l|l|l}
\hline Patologia & $\mathbf{N}^{\mathbf{0}}$ & $\mathbf{\%}$ \\
\hline DMH & 53 & 42,7 \\
\hline Pneumonia & 16 & 12,9 \\
\hline Sepse & 32 & 25,8 \\
\hline Pneumotórax & 4 & 3,2 \\
\hline $\begin{array}{l}\text { Insuficiência } \\
\text { Respiratória }\end{array}$ & 18 & 14,5 \\
Especificada & & \\
\hline Atelectasia & 1 & 0,8 \\
\hline Total & 124 & 100,0 \\
\hline
\end{tabular}

Fonte: autoria própria.

O tempo de permanência em VM foi variado; $50,8 \%$ dos pacientes permaneceram entre 1 a 5 dias em VM. (Tabela 4) 
Tabela 4: Tempo de permanência em VM.

\begin{tabular}{l|l|l}
\hline Tempo de Ventilação & $\mathbf{N}^{\mathbf{0}}$ & $\%$ \\
\hline 1 - 5 Dias & 63 & 50,8 \\
\hline 6 a 10 dias & 44 & 35,5 \\
\hline 11 a 20 dias & 12 & 9,7 \\
\hline$>$ 21 dias & 5 & 4 \\
\hline Total & 124 & 100,0 \\
\hline
\end{tabular}

Fonte: autoria própria.

Referente ao número de neonatos que utilizaram corticoide previamente a extubação foi encontrado 47 pacientes $(37,9 \%)$ (Tabela 5). Na amostra não foi encontrado relação estatística ao se analisar a relação do uso ou não de corticoide antes da extubação com falhas de extubação.

Em estudos mundiais o uso de corticoide não possui impacto na redução no número de falhas de extubação em RN (HEULLIT, GERHARD, ARNOLD, 2008), porém de acordo com Hallyday et al (2010), o uso de corticosteroide pós-natal melhora a função pulmonar, diminuindo a incidência de doença pulmonar crônica futura. Para Markovitz e Randolph (2002) e Lukkassen et al (2006), o uso de corticoide é importante para diminuir os riscos de estridor pós-extubação. Para Garros et al (2001), o uso de corticoide é eficaz na prevenção e no tratamento de laringite pós extubação, quando sua utilização é feita 24 horas antes e mantido até 24 horas após o procedimento.

Tabela 5: Uso de corticoide prévio a extubação dos RN.

\begin{tabular}{l|l|l}
\hline Uso de corticoide & $\mathbf{N}^{\mathbf{0}}$ & $\mathbf{\%}$ \\
\hline Sim & 47 & 37,9 \\
\hline Não & 76 & 61,3 \\
\hline Sem informação & 1 & 0,8 \\
\hline Total & 124 & 100,0 \\
\hline
\end{tabular}

\subsection{ANÁLISE BIVARIADA}

Foi observado um menor número de falhas de extubação no período de implantação e no período de consolidação do protocolo quando comparado com o período onde se fazia o procedimento de forma empírica (período antes da implantação do protocolo) (p-valor 0,038). (Tabela 6) 
Dados estes que se assemelham aos obtidos pelo estudo de Andrade et al (2010). Em um estudo observacional, longitudinal e prospectivo com 60 RNPT, que foram divididos em dois grupos de $30 \mathrm{RN}$. Em um grupo foi aplicado o Teste de Respiração Espontânea e comparado com o outro grupo (controle). Observou - se uma taxa de sucesso de extubação de $66.7 \%$ no grupo em que foi aplicado o TRE contra $36,7 \%$ de sucesso no grupo controle.

Tabela 6: número de falhas de extubação de acordo com os períodos do estudo.

\begin{tabular}{l|l|l|l|l|l}
\hline \multirow{2}{*}{ Implantação } & \multicolumn{3}{|l|}{ Falha de Extubação } & \multirow{2}{*}{ p-valor } \\
\cline { 2 - 5 } & \multicolumn{2}{|l|}{ Sim } & \multicolumn{2}{l|}{ Não } & \\
\cline { 2 - 5 } & $\mathbf{N}^{\mathbf{0}}$ & $\%$ & $\mathbf{N}^{\mathbf{0}}$ & $\%$ & \\
\hline $\begin{array}{l}\text { Antes da } \\
\text { implantação }\end{array}$ & 6 & 42,86 & 21 & 19,09 & \\
\hline $\begin{array}{l}\text { Ano de } \\
\text { Implantação }\end{array}$ & 3 & 21,43 & 15 & 13,64 & \multirow{2}{*}{0,038351} \\
\hline Consolidação & 5 & 35,71 & 74 & 67,27 & \\
\hline Total & 14 & 100 & 110 & 100 & \\
\hline
\end{tabular}

Fonte: autoria própria.

A relação entre a idade gestacional do RN com falhas de extubação não mostrou significância estatística; mas foi observado que $77 \%$ das falhas de extubação ocorreu em RN prematuros, sendo que 43\% ocorreu em RNPT extremo e RNPT muito prematuro (Tabela 7). Fávero et al (2011) descreveu que quanto menor a idade gestacional maior a taxa de falha de extubação e reintubação em RN, fato este explicado pela imaturidade anatômico-fisiológica do trato respiratório (OLIVEIRA, MORAN, 2009), o que gera uma maior instabilidade da caixa torácica e das vias aéreas superiores, consequentemente maior dificuldade na extubação. (HERMETO, BOTTINO, VAILLANCOURT et al, 2009)

Tabela 7: relação entre a idade gestacional e falhas de extubação.

\begin{tabular}{l|l|l|l|l|l}
\hline \multirow{2}{*}{$\begin{array}{l}\text { Idade } \\
\text { Gestacional }\end{array}$} & \multicolumn{4}{|l|}{ Falha de Extubação } & \multirow{2}{*}{ p-valor } \\
\cline { 2 - 5 } & \multicolumn{2}{|l|}{ Sim } & \multicolumn{2}{l}{ Não } & \\
\cline { 2 - 5 } & $\mathbf{N}^{\mathbf{0}}$ & $\%$ & $\mathbf{N}^{\mathbf{0}}$ & $\%$ & \\
\hline RNT & 3 & 21,43 & 14 & 12,73 & \\
\hline RN Pós Termo & 0 & 0,00 & 1 & 0,91 & \multirow{2}{*}{0,276836} \\
\hline RNPT tardio & 5 & 35,71 & 25 & 22,73 & \\
\hline RNPT Extremo & 2 & 14,29 & 26 & 23,64 & \\
\hline
\end{tabular}




\begin{tabular}{|c|c|c|c|c|}
\hline $\begin{array}{l}\text { Muito } \\
\text { prematuro }\end{array}$ & 4 & 28,57 & 44 & 40,00 \\
\hline Total & 14 & 100 & 110 & \begin{tabular}{|l|l|}
100 \\
\end{tabular} \\
\hline
\end{tabular}

Fonte: autoria própria.

Quando se observa as falhas de extubação relacionando-as ao peso de nascimento, temos que 71,4\% (N:10) dos RN que apresentaram falha de extubação eram de baixo peso, mas esses dados não apresentaram significado estatístico na relação dessas duas variáveis. (Tabela 8)

Tabela 8: relação do peso de nascimento com falhas de extubação

\begin{tabular}{|c|c|c|c|c|c|}
\hline \multirow{3}{*}{$\begin{array}{l}\text { Peso ao } \\
\text { nascer }\end{array}$} & \multicolumn{4}{|c|}{ Falha de Extubação } & \multirow{3}{*}{ p-valor } \\
\hline & \multicolumn{2}{|c|}{ Sim } & \multicolumn{2}{|l|}{ Não } & \\
\hline & $\mathbf{N}^{\mathbf{0}}$ & $\%$ & $\mathbf{N}^{\mathbf{0}}$ & $\%$ & \\
\hline$<1000 \mathrm{~g}$ & 2 & 14,29 & 19 & 17,27 & \\
\hline $\begin{array}{l}1000 \quad \mathrm{a} \\
1500 \mathrm{~g}\end{array}$ & 3 & 21,43 & 31 & 28,18 & \\
\hline $\begin{array}{l}1500 \quad \mathrm{a} \\
2500 \mathrm{~g}\end{array}$ & 5 & 35,71 & 41 & 37,27 & 0,768656 \\
\hline $\begin{array}{ll}2500 \quad a \\
3800 g\end{array}$ & 4 & 28,57 & 19 & 17,27 & \\
\hline Total & 14 & 100 & 110 & 100 & \\
\hline
\end{tabular}

Fonte: autoria própria.

Porém, como evidenciado nos estudos de Dimitriou et al (2002) e Hermeto et al (2009), observou-se que um dos fatores de falha na extubação está relacionado com o baixo peso do RN, principalmente naqueles com peso menor que 1250 gramas, devido a imaturidade da musculatura acessória nessa faixa de peso.

A patologia que mais se relacionou com as falhas de extubação foi a DMH, o que é compatível com o perfil da idade gestacional e o peso dos RN internados na UTI. (Tabela 9)

Tabela 9: relação entre as patologias dos RN com as falhas de extubação.

\begin{tabular}{l|l|l|l|l|l}
\hline \multirow{4}{*}{ Patologia } & \multicolumn{3}{|l|}{ Falha de Extubação } & \multirow{2}{*}{ p-valor } \\
\cline { 2 - 5 } & Sim & \multicolumn{2}{|l|}{ Não } & \\
\cline { 2 - 5 } & $\mathbf{N}^{\mathbf{0}}$ & $\%$ & $\mathbf{N}^{\mathbf{0}}$ & $\%$ & \\
\hline
\end{tabular}




\begin{tabular}{|c|c|c|c|c|c|}
\hline DMH & 4 & 28,57 & 49 & 44,55 & \multirow{7}{*}{0,084741} \\
\hline Pneumonia & 3 & 21,43 & 13 & 11,82 & \\
\hline Sepse & 4 & 28,57 & 28 & 25,45 & \\
\hline Pneumotórax & 2 & 14,29 & 2 & 1,82 & \\
\hline $\begin{array}{l}\text { Insuficiência } \\
\text { Respiratória } \\
\text { Não } \\
\text { Especificada }\end{array}$ & 1 & 7,14 & 17 & 15,45 & \\
\hline Atelectasia & 0 & - & 1 & 0,91 & \\
\hline Total & 14 & 100 & 110 & 100 & \\
\hline
\end{tabular}

Fonte: autoria própria.

Não foi encontrada relação estatística entre a falha de extubação com o tempo de VM que a que foi submetido o RN. (Tabela 10)

Tabela 10: relação entre o tempo de VM com falhas de extubação.

\begin{tabular}{|c|c|c|c|c|c|}
\hline \multirow{3}{*}{ Tempo de VM } & \multicolumn{4}{|c|}{ Falha de Extubação } & \multirow{3}{*}{ p-valor } \\
\hline & \multicolumn{2}{|c|}{ Sim } & \multicolumn{2}{|l|}{ Não } & \\
\hline & $\mathbf{N}^{\mathbf{0}}$ & $\%$ & $\mathbf{N}^{\mathbf{0}}$ & $\%$ & \\
\hline $1-5$ Dias & 4 & 28,57 & 59 & 53,64 & \\
\hline 6 a 10 dias & 8 & 57,14 & 36 & 32,73 & \\
\hline 11 a 20 dias & 2 & 14,29 & 10 & 9,09 & 0,276018 \\
\hline$>=21$ dias & 0 & - & 5 & 4,55 & \\
\hline Total & 14 & 100 & 110 & 100 & \\
\hline
\end{tabular}

Fonte: autoria própria. 
No grupo selecionado foi encontrado forte relação entre a falha do TRE com as falhas de extubação. (Tabela 11):

Tabela 11. Relação entre a falha no TRE com falhas de extubação

\begin{tabular}{|c|c|c|c|c|c|}
\hline \multirow{3}{*}{$\begin{array}{l}\text { Teste de } \\
\text { Respiração } \\
\text { Espontânea }\end{array}$} & \multicolumn{4}{|c|}{ Falha de Extubação } & \multirow{3}{*}{ p-valor } \\
\hline & \multicolumn{2}{|c|}{ Sim } & \multicolumn{2}{|c|}{ Não } & \\
\hline & $\mathbf{N}^{\mathbf{0}}$ & $\%$ & $\mathbf{N}^{\mathbf{0}}$ & $\%$ & \\
\hline Sim & 6 & 42,86 & 89 & 80,91 & \\
\hline Não & 8 & 57,14 & 21 & 19,09 & (0,001555 \\
\hline Total & 14 & 100 & 110 & 100 & \\
\hline
\end{tabular}

Fonte: autoria própria.

Não foi encontrada relação entre as pressões e o Pico de Pressão com as falhas de extubação. (Tabela1)

Tabela 12: relação entre o Pico de Pressão com falhas de extubação.

\begin{tabular}{|c|c|c|c|c|c|c|}
\hline \multirow{3}{*}{$\begin{array}{l}\text { Pico } \\
\text { Pressão }\end{array}$} & \multirow{3}{*}{ de } & \multicolumn{4}{|c|}{ Falha de Extubação } & \multirow{3}{*}{ p-valor } \\
\hline & & \multicolumn{2}{|c|}{ Sim } & \multicolumn{2}{|l|}{ Não } & \\
\hline & & $\mathbf{N}^{\mathbf{0}}$ & $\%$ & $\mathbf{N}^{\mathbf{0}}$ & $\%$ & \\
\hline 9 a 15 & & 13 & 92,86 & 107 & 97,27 & \\
\hline 16 a 20 & & 1 & 7,14 & 3 & 2,73 & 0,378468 \\
\hline Total & & 14 & 100 & 110 & 100 & \\
\hline
\end{tabular}

\section{CONCLUSÃO}

O desmame da ventilação mecânica é um desafio em Unidade de Terapia Intensiva Neonatal e está vinculada ou sujeita a complicações que podem levar a um aumento da mortalidade dentro da UTIN.

A VM é um procedimento necessário para a sobrevivência, mas não é isento de riscos, causando lesões ao pulmão doente ou imaturo. Em neonatos, o uso da VM por tempo excessivo 
e as vezes desnecessárias pode levar a várias complicações como: infecção, displasia broncopulmonar, maior risco de morte, pior prognóstico no desenvolvimento neuropsicomotor. Retirar o paciente da VM pode ser mais difícil que mantê-lo. O processo de retirada do suporte ventilatório ocupa ao redor de $40 \%$ do tempo total de ventilação mecânica.

O processo de retirada da VM, na grande maioria dos serviços de terapia intensiva neonatal, ainda está sujeito a condutas pouco embasadas cientificamente.

Sob o ponto de vista profissional, criar mecanismos facilitadores na UTI pode abreviar o tempo de VM; visto que os profissionais devem se sentir mais seguros ao identificar o paciente capaz de se submeter ao teste de respiração espontânea (TRE) diminuindo, assim, o grau de insucesso das extubações, garantindo melhor evolução do paciente.

Podemos concluir então no nosso estudo que o sucesso da extubação está intimamente ligado com a aplicação do TRE, sendo este um mecanismo necessário para uma evolução satisfatória dos pacientes da nossa UTIN. Fica assim a sugestão no sentido de que as Unidades de Saúde venham a adotar um Protocolo especifico com o fim de reduzir as taxas de falhas de extubação em neonatos e suas indesejáveis complicações. 


\section{REFERÊNCIAS}

1. ANDRADE, L.B. et al. Avaliação do teste de respiração espontânea na extubação de neonatos pré-termo. RevBras Ter Intensiva. São Paulo, v.22, n.2, p159-165. 2010.

2. ANTUNES, L.C.O. Desmame da Ventilação Mecânica e Extubação no Recém-nascido. In: ASSOCIAÇÃO BRASILEIRA DE FISIOTERAPIA CARDIORRESPIRATÓRIA E FISIOTERAPIA INTENSIVA. PROFISIO Programa de Atualização em Fisioterapia Pediátrica e Neonatal: Cardiorespiratória e Terapia Intensiva. Ciclo 1. Porto Alegre: Artmed Panamericana, 2012 v. 1, n. 1, p. 83-105.

3. AZEREDO, C. A. C. Técnicas para o Desmame no Ventilador Mecânico. São Paulo: Manole, 2002.

4. BOUSSO, A. et al. Evaluation of the dead space to tidal volume ratio as a predictor of extubation failure. Journal of Pediatrics, v. 82, p. 347-353, 2008.

5. COLOMBO, T. et al. Implementação, avaliação e comparação dos protocolos de desmame com tubo $\mathrm{T}$ e pressão suporte associada à pressão expiratória final positiva em pacientes submetidos à ventilação mecânica por mais de 48 horas em unidade de terapia intensiva. Revista Brasileira de Terapia intensiva, vol.19, n1, jan-mar.2007.

6. DANAGA, A.R, et al. Avaliação do desempenho diagnóstico e do valor de corte para o índice de respiração rápida e superficial na predição do insucesso da extubação. Jornal Bras. Pneumol, São Paulo, 2009.

7. DIMITRIOU, G.; GREENOUGH, A.; ENDO, A. et al. Prediction of extubation failure in preterm infants. Arch Dis Child Fetal Neonatal Ed. 2002 Jan; v.86, n.1, F.32-35, Jan. 2002

8. ESTEBAN, A.; ALÍA, I.; IBAÑES, J. et al. Modes of mechanical ventilation and weaning. A national survey of Spanish hospitals. The Spanish Lung Failure $\begin{array}{llll}\text { Collaborative } & \text { Group. } & \text { Chest. } & 1994 ; 106(4): 1188-93 .\end{array}$ PMid:7924494. http://dx.doi.org/10.1378/chest.106.4.1188

9. FÁVERO, R.A.; SCHUSTER, R.C.; WOJAHN, V.W.; TARTARI, J.L. Incidência e principais fatores associados à falha na extubação em recém-nascidos prematuros. Pediatria São Paulo. v.33,n.1, p.13-20, 2011.

10. GARROS, D. laringite pós-extubação respirando aliviados. Jornal de Pediatria.v.77, n.3, p.157-159, 2001. 
11. GOLDSMITH, J.P; SHARP, M.J. Ventilatory management casebooks. In: GOLDSMITH, J.P.; KAROTKIN, E.H (Ed.). Assisted ventilation of the neonate. 2nd. Ed. Philadelphia: WB Saunders Company; 1988, p. 409-429.

12. GOLDWASSER, R.; FARIAS, A. Desmame e Interrupção da Ventilação Mecânica. In: Consenso Brasileiro de Ventilação Mecânica, 3, 2007, Brasília. Jornal Brasileiro Pneumologia, v.33, Supl.2, p. $128-136$.

13. Goldwasser, R.S.; David, C.M. Desmame da Ventilação Mecânica: Promova uma Estratégia. Revista Brasileira de Terapia Intensiva, v. 19, n. 1, p. 107-112, Jan.-Mar. 2007.

14. GONÇALVES, J.Q.et al. Características do processo de desmame da ventilação mecânica em hospitais do distrito federal. Revista Brasileira de Terapia Intensiva, v.19, n. 1, Jan-Mar. 2007.

15. HALLIDAY, H.L.; EHRENKRANZ, R.A.; DOYLE, L.W. Early ( $<8$ days) postnatal corticosteroids for preventing chronic lung disease in preterm infants. Cochrane Database Syst Rev. 2010;(1): CD001146.

16. HARRISON, A.M. et al. Failed extubation after cardiac surgery in Young children: prevalence, pathogenesis, and risk factors. Pediatric Critical Care Medicine, v. three, n.2, p. 148-152, 2002.

17. HERMETO, F. et al. Incidência e principais fatores associados à falha de extubação em recém-nascidos com peso de nascimento $<1.250$ gramas. Jornal de Pediatria, Porto Alegre, v. 85, n. 5, Out. 2009.

18. HERMETO, F.; BOTTINO, M.N.; VAILLANCOURT, K. et al. Implementation of a respiratory therapist-driven protocol for neonatal ventilation: impact on the premature population. Pediatrics. v.123,n.5, p.907-916, 2009.

19. HERMETO, F.; MARTINS, B.M.; RAMOS, J.R. et al. Incidence and main risk factors associated with extubation failure in newborns with birth weight $<1,250$ grams. J Pediatr. Rio J, 2009 Sep-Octv.85, n.5, p. 397-402, sep/oct. 2009.

20. HEULliT, M.J.; GERHARD, H, W, ARNOLD, J.H. Mechanical Ventilation. In: LIPPINCOT, W.; WILKINS. Rogers' Textbook of Pediatric Intensive. Fourth Edition. Philadelphia 2008; p. 508-531.

21. KARCZ, M. et al. State of the art Mechanical Ventilation.JOURNALOFCARDIOTHORACICANDVASCULARANESTHESIA, May. 2011. 
22. LUKKASSEN, I.M.; HASSING, M.B.; MARKHORST, D.C. Dexamethasone reduces reintubation rates due to postextubation stridor in a high risk paediatric population. Acta Paediatr. v.95, p.74-76, 2006.

23. MARKOVITZ, B.P.; RANDOLPH, A.G. Corticosteroids for prevention of reintubation and postextubation stridor in paediatric patients: A meta-analisys. Pediatric Critical Care Medicine.v.3, p.222-226, 2002.

24. OLIVEIRA, C.H.; MORAN, C.A. Estudo descritivo: ventilação mecânica não invasiva em recém-nascidos pré-termo com síndrome do desconforto respiratório. ConScientiae Saúde.v.8, n.3, p.485-489, 2009.

25. PEREIRA, K.D; SMITH, S.L; HENRY, M. Failed extubation in the neonatal intensive care unit. InternationalJournalofPediatricOtorhinolaryngology, vol. 71, n. 11, p. 1763-1766, 2007.

26. SANTOS, E.S.M; OLIVEIRA, A.C.T; BERENCHTEIN, B. Protocolo de desmame em Neonatologia. UNILUS Ensino e Pesquisa, v. 11, n. 24, p. 31, 2014.

27. STOLL, B.J. et al. Neonatal outcames of extremely preterm infants from the NICHD Research Network. Pediatrics, v. 126, n.3, p. 443-456, Sep. 2010.

28. TEIXEIRA, C.; MACCARI, J.G.; VIEIRA, S.R. et al. Impacto de um protocolo de desmame de ventilação mecânica na taxa de falha de extubação em pacientes de difícil desmame. J Bras Pneumol. v.38, n.3, p.364-37, Maio/jun. 2012.

29. ULSENHEIMER, M.M.M. Avaliação de um protocolo de desmame da ventilação mecânica em crianças.2006.Dissertação(Mestrado em Pediatria). Ciências da Saúde, UFP, Curitiba, 2006. 


\section{ANEXOS}

ANEXO 1

CHECK LIST DE EXTUBAÇÃO PROGRAMADA (ANEXO1)

DATA

PACIENTE:

FISIOTERAPEUTA

RESPONSÁVEL:

MÉDICO

RESPONSÁVEL:

\begin{tabular}{|l|l|}
\hline ITENS & CHECAGEM \\
\hline FiO2 < 40\% & \\
\hline PEEP 5 & \\
\hline FR espontânea > 40 rpm & \\
\hline Falhas de extubação anteriores & \\
\hline Uso de corticoide, em caso de falhas de extubação anterior & \\
\hline Estabilidade hemodinâmica & \\
\hline Redução / Retirada de sedoanalgesia & \\
\hline Relação PaO2 / FiO2 > 200 & \\
\hline Jejum prévio de 3 horas & \\
\hline Acidose metabólica, acidose respiratória ou retenção de CO2 & \\
\hline Fisioterapia respiratória (Higiene brônquica) & \\
\hline $\begin{array}{l}\text { TRE } \\
\text { / } \uparrow 2000 \text { gr }=1000 \text { gr=4min / 1000gr-1500gr=5min / 1500-2000gr=5 a 10min }\end{array}$ & \\
\hline
\end{tabular}

OBS.: 


\section{ANEXO 2}

\section{FICHA DE COLETA DE DADOS}

1) Ano de nascimento:

2) Idade gestacional ao nascimento

3) Idade gestacional corrigida no momento da extubação

4) Peso ao nascimento

5) Classificação do RN

6) APGAR

7) Patologia que levou a indicação de VM

8) Tempo de VM

9) Uso de corticoide

10) Fração inspirada de Oxigênio (FiO2),

11) Pressão de pico (PP)

12) Frequência Respiratória no momento da extubação (FR),

13) Realização ou não do teste de respiração espontânea (TRE),

14) Falha de extubação

15) SNAP PE II 